Dr. von Ramer ist der Ansicht, daß die Verwendung anderer Mehle nur entweder allgemein zu erlauben, oder allgemein zu verbieten sei.

Dr. Lührig bittet nochmals die unhaltbare Grenzzahl von $45 \mathrm{mg}$ Lecithinphosphorsäure fallen zu lassen; es könne dies unbeschadet geschehen, da die Begutachtung ja doch dem Einzelnen überlassen bleibe.

Dr. Juckenack bittet damit noch zu warten.

Dr. Sendtner ersucht ebenfalls, mit dem Abändern dieser Grenzzabl zu warten; wir werden nicht Gefahr laufen, deshalb ungerecht beurteilt $\mathrm{zu}$ werden, oder andere ungerecht zu beurteilen, wenn wir auch dem Ätherextrakt Beachtwng schenken. Die ausländischen Marktwaren dürften im allgemeinen allerdings nicht unter die vom Verbande der Teigwarenfabrikanten aufgestellte Definition fallen, jedoch sei daran zu erinnern, daß z. B. Makkaroni gewöhnlich aus Weich- und Hartgries von Weizen hergestellt würden.

Der Vorsitzende beantragt, die bisherige Grenzzahl von $45 \mathrm{mg}$ Lecithinphosphorsäure solange aufrecht zu erhalten, bis weitere Erfahrungen vorliegen.

Dieser Antrag wird einstimmig angenommen.

Dr. W. Fresenius glaubt, daB die von S end th er gegebene Definition eine generelle sei; es frage sich, ob sie so allgemein angenommen werden könne.

H. Trillich bemerkt, daßs wir den Italienern keine besondere Stellung einräumen dürften; die dentsehe Industrie könne auch einmal in die Lage kommen, andere Mehle verwenden zu müssen; die Mehlfrage sei daher auszuschlieken, weil dadurch die Industrie geschädigt werde. stellen.

Dr. B öm er hält diesen Punkt ebenfalls für nicht spruchreif und bittet ihn zurückzu-

Der Vorsitzende beantragt die Rohmaterialfrage zurückzustellen.

Dieser Antrag wird angenommen.

\title{
Zur Kenntnis der im rechtsdrehenden Koniferenhonig vorkommenden Dextrine.
}

Von

Dr. A. Hilger in München.

Die Tatsache, daß wir noch nicht in der Lage sind, die rechtsdrehenden Dextrine des Koniferenhonigs, nicht minder auch die Dextrine der linksdrehenden Honige chemisch mit Sicherheit zu charakterisieren, ebensowenig deren Vergärbarkeit festzustellen, gab Veranlassung zu einer eingehenden Versuchsreihe, welche von Herrn Dr. Paul Wolff durchgeführt wurde und deren Zweck es war, die in typischen rechtsdrehenden Koniferenhonigen vorkommenden Dextrine chemisch zu charakterisieren und deren Vergärbarkeit festzustellen.

O. Haenle $\mathrm{e}^{1}$ ) hat zuerst durch Untersuchungen festgestellt, daß auch rechtsdrehende Honige zweifellos als echt angesehen werden nüssen, während die meisten Blütenhonige den polarisierten Lichtstrahl nach links drehen, daß ferner in an Koniferen reichen Gegenden rechtsdrehende Tannenhonige hänfiger vorkommen und als die Ursache dieser Rechtsdrehung ein dextrinähnliches Kohlenhydrat angesehen werden muB.

E. v. Raume $\mathbf{r}^{2}$ ) und W. Mader ${ }^{3}$ ) suchten das Dextrin nach Vergärung des Honigs durch Bierhefe mit Alkohol zu fällen.

O. Haenle $e^{4}$ und E. v. Raume $\mathbf{r}^{5}$ ) fällten später die Dextrine mit Alkohol-

1) Wigger s u. Husemann, Jahresbericht 1883-1884, 590.

2) Zeitschr. angew. Chem. 1889, 607 .

3) Archiv f. Hygiene 1890, 10, 436.

4) O. Haenle, Die Chemie des Honigs. Strakburg 1892.

5) Zeitschr. angew. Chem. 1889, 607. 
Wassermischungen durch Fraktionieren aus dem Honig; sie waren aber nicht in der Lage reine Körper zu erhalten, welche sich charakterisieren ließen.

K. Amthor ${ }^{1}$ ) versuchte die Dextrine aus dem vergorenen Honig durch Eindampfen rein $\mathrm{zu}$ erhalten.

Die von den genannten Forschern erhaltenen Dextrine lieferten bei der Hydrolyse Glykose.

O. Künnmann und A. Hilge $\mathbf{r}^{2}$ ) beschäftigten sich ebenfalls mit der Isolierung von Dextrin aus rechtsdrehendem Honig sowie auch mit den Gärungserscbeinungen bei Finwirkung verschiedener Reinkulturhefen. Sie vermuten, daß die Rechtsdrehungen verschiedener Naturhonige durch die Gegenwart von Achroodextrin, dem noch Maltose beigemegt ist, veranlaßt werden.

E. Bekmann ${ }^{3}$ ) mit seinen Schülern E. Burkhardt $t^{4}$, J. Monheim ${ }^{5}$ ) und A. Mülle $\mathbf{r}^{n}$ ) setzten die Studien über die Honigdextrine fort.

Sie untersuchten das Verhalten von Stärke-Dextrinen, Stärkesyrup, festem Stärkezucker, Koniferenhonig und Gemischen davon gegen Methyl- und Äthylalkohol mit Baryumhydratlösung bezw. Bleiessig. Als Ergebnis dieser Arbeiten wurde festgestellt, „daß das in rechtsdrehenden Honigen enthaltene Dextrin ein niedriges Molekulargewicht besitzt", ferner „daß die starke Rechtsdrehung des untersuchten Tannenhonigs nur zum geringsten Teil deu spärlich vorhandenen Dextrinen zuzuschreiben ist.

Außerdem wird ausgesprochen, „daß das rechtsdrehende Prinzip des untersuchten Tannenhonigs, das sogenannte Honigdextrin, keineswegs mit einem Stärkedextrin identisch ist, daf es vielmehr ein Disaccharid darstellt, verschieden von Maltose und doch durch Hydrolyse in Glykose überführbar. Es wurde der Benzoylester des Honigdextrins dargestellt, ebenso der Sulfonsäureester.

In nenester Zeit stellten O. Haenle und A. Scholz ${ }^{7}$ ) auf Grund einer Arbeit von M. Hönig ${ }^{8}$ ) Versuche an, diese Metbode zur Trennung der im Honig vorkommenden Dextrine anzuwenden; sie zeigten, daß das Dextrin der Tannenhonige aus verschiedenen schwer vergärbaren Körpern besteht und deshalb durch Vergärung des Honigs mit Weinhefe zu erhalten ist.

Haenle und Scholz stellten das Dextrin aus der vergorenen Honiglösung durch Fällung mit Alkohol-Äther her, bestimmten ferner das Reduktionsvermögen dieses Dextrins gegen F e h ling'sche Lösung direkt, nach halbstündiger Inversion mit 1\%-iger Salzsäure und nach dreistündiger Inversion mit konzentrierter Salzsäure unter Druck auf dem Wasserbade.

Nach diesen Versuchen ist das Reduktionsvermögen gegen Fehling'sche Lösung sehr gering. Durch Inversion werden aber etwa $30 \%$ in ein reduzierendes Kohlenhydrat verwandelt. Ein Teil dieses Dextrins ist in $87 \%$-igem Alkohol unlöslich und aus verdünntem Alkohol als Baryumverbindung fällbar.

1) Vierteljahressehrift Nahrungs- u. Genufmittel 1887, 2, 409.

2) Forschungsberichte über Lebensmittel 1896, 3, 211 .

3) Zeitschr. analyt. Chem. 1896, 35, 203.

*) E. Burkh ardt, Inangural-Dissertation. Erlangen 1897.

5) J. Mohnheim, Inaugural-Dissertation. Erlangen 1899.

8) Diese Zeitschrift 1901, 4, 1068.

7) Diese Zeitschrift 1908, 6, 1027.

8) Diese Zeitschrift 1902, 5, 641. 


\section{Eigene Versuche zur Abscheidung und Kennzeichnung der Honigdextrine.}

$\mathrm{Zu}$ den eigenen Arbeiten übergehend, ist vor allem zu betonen, daß vier typische Koniferenhonige mit starker Rechtsdrehung als Rohmaterialien für die Untersuchung verwendet wurden und zwar:

Zwei Koniferenhonige aus Baden (Kinzigtal 1902 und Murgtal 1903), ein Honig aus Oberelsap (1903),

ein Honig aus Wasserburg am Bodensee (1903).

Un das rechtsdrehende Prinzip zu isolieren, gingen wir von Fällungen mit Methylalkohol aus. Diese wurden so ausgeführt, daß der Honig durch Erwärmen verflüssigt, mit der gleichen Menge Methylalkohol angerieben und schließlich so viel desselben Alkohols hinzugebracht wurde, daß im ganzen die zehnfache Menge des Honigs angewendet wurde. Das Gemisch blieb zunächst ziemlich klar, aber nach einiger Zeit schied sich eine schmutziggelbe Masse in Flocken ab, die durch Absitzenlassen und Abhebern der überstehenden Flüssigkeit gesammelt wurde. Ein Abfiltrieren der Masse war nicht möglich, da sich die Poren des Filtrierpapiers bald zusetzten, so daß kein Tropfen der Flüssigkeit mehr ablief. Dagegen hatte der Versuch, durch Zentrifugieren den Körper von der Flüssigkeit zu trennen, Erfolg. Auf diese Weise ließ sich die Masse auch auswaschen, indem man sie wiederholt mit Methylalkohol anrieb und wiederum durch Zentrifugieren Fällung und Flüssigkeit trennte. Schließlich wurde die feuchte Masse im Vakuum über Phosphorpentoxyd getrocknet.

Der so erhaltene Körper stellte ein braungelbes Pulver dar, das, einmal trocken, nicht mehr hygroskopisch war. In Wasser war es nur zur Hälfte löslich; es blieb eine grauschwarze Masse zurück, die nur insoweit näher untersucht wurde, als durch die gewöhnliche Reaktion ein beträchtlicher Gehalt an Stickstoff nachgewiesen wurde.

Die wässerige Lösung sollte nun das gesuchte Honigdextrin enthalten, dessen Abscheidung auch wieder durch Fällen mit Methylalkohol, Zentrifugieren und Trocknen über Phosphorpentoxyd gelang.

Der Körper löste sich jetzt vollkommen in Wasser, aber mit braunroter Farbe; diese verhinderte festzustellen, $o b$ hier der die Ebene des polarisierten Lichtes nach rechts ablenkende Körper vorlag oder nicht. Es muBte daher vor allem daran gelegen sein, die Lösung möglichst zu entfärben. Ein Niederschlagen des Farbstoffes mit Tonerdehydrat mißlang, ebenso wurde durch Aufkochen mit frisch ausgeglühter Tierkohle wenig erreicht. Bei einem Versuch, den Farbstoff mit neutralem Bleiacetat zu fällen, trat eine starke Ausscheidung ein. Es lag deshalb die Vermutung nahe, daß dureh neutrales Bleiacetat nicht nur der Farbstoff, sondern auch der gesuchte Körper gefällt würde. Der so gebildete Niederschlag wurde gesammelt und mit Schwefelwasserstoff zerlegt.

Das Filtrat vom gebildeten Bleisulfid wurde durch Eindampfen konzentriert und wieder mit der zehnfachen Menge Methylalkohòl versetzt. Es schied sich dieses Mal ein schön weißer Körper in Flocken aus, der nach dem Trocknen ein rein weißes, nicht hygroskopisches Pulver darstellte. Er war nun in Wasser mit hellgelber Farbe vollkommen löslich; seine Lösung drehte aber weder den polarisierten Lichtstrahl, noch reduzierte sie nach dem Kochen mit verdünnter Salzsäure F e hling'sche Lösung. Es mußte also hier etwas anderes, als das gesuchte Honigdextrin vorliegen. Eine Elementaranalyse lieferte immer schwankende Werte, da der Aschengehalt äber $50 \%$ betrug. Dennoch zeigte sie, daß ein organischer Körper vorliegen mußte. Auf Grund 
der Fällbarkeit mit neutralem Bleiacetat wurde deshalb eine größere Menge des Körpers dargestellt, und zwar so, daß nunmebr die wässerige Honiglösung direkt der Fällung unterzogen wurde.

$\mathrm{Zu}$ diesem Zwecke wurde $1 \mathrm{~kg}$ Honig in $2 \mathrm{~kg}$ Wasser gelöst und eine wässerige Lösung von $12 \mathrm{~g}$ Bleiacetat hinzugefügt. Es entstand sofort ein flockiger, gelbbrauner Niederschlag, der sich rasch absetzte. Auf Zusatz von weiterem Bleiacetat zu einer Probe der überstehenden Flüssigkeit entstand kein Niederschlag mehr, ein Zeichen, daß alles ausgefällt war. Am besten gelang es, den Niederschlag von der zuckerhaltigen, überstehenden Flüssigkeit durch häufiges Dekantieren zu trennen. Die Entfernung des Zuckers in der überstehenden Flüssigkeit war nach fünfmaligem Dekantieren eingetreten. Der Niederschlag wurde schlieBlich in Wasser fein verteilt und durch Schwefelwasserstoff zerlegt; dabei blieb viel Bleisulfid in der Flüssigkeit kolloidal gelöst, welches sich auch durch Erwärmen nicht zur Abscheidung bringen lief. Dagegen gelang dieses durch einstündiges Erwärmen mit etwas Ammoniumnitrat.

Das eingedampfte Filtrat lieferte auf Zusatz von Methylalkohol eine weiße flockige Fällung. Aber auch bei dieser Darstellung wurde ein stark aschenhaltiger Körper erhalten, der zur Elementaranalyse nicht tauglich war.

Es mufte daher das Bestreben sein, den Körper durch irgend eine Methode möglichst aschenfrei darzustellen. Die Asche bestand aus Calcium- und Magnesiumphosphat und reagierte auBerdem alkalisch. Es wurde eine nochmalige Fällung mit Bleiacetat in salpetersaurer Jösung versucht, aber auch hierdurch wurde keine Verminderung der Mineralbestandteile erreicht, vielmehr war der Versuch mit großen Verlusten an Substanz verbunden. Auch durch wiederholtes Fällen mit Methylalkohol war keine Verminderung, sondern im Gegenteil eine Amreicherung an Mineralbestandteilen zu verzeichnen.

So wurde denn ein anderer Versuch gemacht, der darin bestand, einen der Mineralbestandteile, nämlich die Phosphorsäure, zu entfernen, um dadurch die Bedingungen wegzunehmen, daß sich beim Fällen der Phosphorsaure Kalk mit dem Körper niederschlagen konnte. Diese Methode hatte Erfolg.

Vor der Entfernung der Phosphorsäure mußte übrigens etwa vorhandene Ameisensäure durch Destillieren der wässerigen angesäuerten Lösung mit Wasserdampf fortgeschafft werden. Es zeigte sich jedoch, daß keine Ameisensäure mehr vorhanden war; wahrscheinlich war durch das häufige Dekantieren des Bleiniederschlages etwa vorhandenes Ameisensaures Blei in Lösung gegangen. Zur Entfernung der Phosphorsäure wurde die ammoniakalische Lösung mit Magnesiam-Mixtur versetzt und in der Kälte 24 Stunden stehen gelassen. Es schied sich eine reichliche Krystallmasse von Ammonium-Magnesiumphosphat aus.

Die vom Ammonium-Magnesiumphosphat abfiltrierte ammoniakalische Lösung wurde mit Tierkohle entfärbt, mit Essigsäure schwach angesäuert und wieder mit Bleiacetat gefällt. Der Bleiniederschlag wurde durch Dekantieren gewaschen, bis keine Ammoniumsalze mehr in der überstehenden Flüssigkeit nachzuweisen waren. Die darauffolgende Zerlegung mit Schwefelwasserstoff beanspruchte ein längeres Eínleiten eines kräftigen Gasstromes. Die vom Bleisulfid abfiltrierte Lösung blieb auch nach dem Verjagen des Schwefelwasserstoffes sauer, es schien also hier eine organische Säure vorzuliegen. Mit Methylalkohol entstand jetzt keine Fällung mehr, die freie Säure ist also darin löslich. Es gelang nicht, die Säure in krystallisiertem Zustande darzustellen, deshalb wurde versucht, mit Hilfe eines krystallisierbaren Salzes zu er- 
mitteln, welche organische Säure hier vorlag. Dieses gelang durch die Darstellung des Baryumsalzes.

\section{Darstellung des Baryumsalzes.}

Die sauere wässerige Lösung wurde mit frisch gefälltem Baryumkarbonat neutralisiert. Bei dem Verdampfen dieser Lösung schieden sich farblose, schön ausgebildete Krystalle aus, die sich unter dem Mikroskope als zu Büscheln vereinigte Nadeln zeigten.

Die Elementaranalyse des über Chlorcalcium getrockneten Salzes zeigte einen Kohlenstoff- und Wasserstoffgehalt, der mit dem des Äpfelsauren Baryums mit 2 Mol. Krystallwasser übereinstimmte, ebenso entsprach der Baryumgehalt diesem Salze.

1. Analyse des wasserhaltigen Baryumsalzes.

Zur Elementaranalyse wurde das Salz fein gepulvert und im Mischrohr mit Bleichromat gemischt, da sonst das bei der Verbrennung intermediär sich bildende Baryumkarbonat nicht zerlegt worden wäre.

a) Elementaranalyse.

1. $0,1838 \mathrm{~g}$ Substanz lieferten $0,1054 \mathrm{~g}$ Kohlensäure und $0,0480 \mathrm{~g}$ Wasser $=$ $15,64 \%$ Kohlenstoff und $2,92 \%$ Wasserstoff.

2. $0,2046 \mathrm{~g}$ Substanz lieferten $0,1176 \mathrm{~g}$ Kohlensäure und $0,0514 \mathrm{~g}$ Wasser $=$ $15,67 \%$ Kohlenstoff und 2,81\% Wasserstoff.

b) Baryumbestimmung.

1. $0,1950 \mathrm{~g}$ Substanz lieferten $0,1494 \mathrm{~g}$ Baryumsulfat $=45,09 \%$ Baryum.

2. $0,2546 \mathrm{~g}$ Substanz lieferten $0,1942 \mathrm{~g}$ Baryumsulfat $=44,89 \%$ Baryum.

Bexechnet für :

$\mathrm{C}_{4} \mathrm{H}_{4} \mathrm{O}_{5} \cdot \mathrm{Ba}+2 \mathrm{H}_{2} \mathrm{O}$

Baryum $44,91 \%$

Koblenstoff 15,73 ,

Wasserstoff 2,62 ,

\begin{tabular}{cc}
\multicolumn{2}{c}{ Gefunden : } \\
I & II \\
$45,09 \%$ & $44,89 \%$ \\
$15,64 \%$ & $15,67 \%$ \\
$2,92 \%$ & $2,81 \%$
\end{tabular}

2. Analyse des bei $105^{\circ}$ bis zur Gewichtsbeständigkeit getrockneten, wasserfreien Salzes.

a) Elementaranalyse.

1. $0,1606 \mathrm{~g}$ Substanz lieferten $0,1038 \mathrm{~g}$ Kohlensäure und 0,0238 g Wasser = $17,63 \%$ Kohlenstoff und 1,65\% Wasserstoff.

2. $0,2028 \mathrm{~g}$ Substanz lieferten $0,1320 \mathrm{~g}$ Kohlensüure und 0,0348 $\mathrm{g}$ Wasser $=$ $17,75 \%$ Kohlenstoff und $1,91 \%$ Wasserstoff.

b) Baryumbestimmung.

1. $0,1686 \mathrm{~g}$ Substanz lieferten $0,1464 \mathrm{~g}$ Baryumsulfat $=51,10 \%$ Baryum.

2. $0,1714 \mathrm{~g}$ Substanz lieferten $0,1480 \mathrm{~g}$ Baryumsulfat $=50,82 \%$ Baryum. 


\begin{tabular}{|c|c|c|c|}
\hline \multirow{2}{*}{\multicolumn{2}{|c|}{$\begin{array}{c}\text { Berechnet für: } \\
\mathrm{C}_{4} \mathrm{H}_{4} \mathrm{O}_{5} . \mathrm{Ba}\end{array}$}} & \multicolumn{2}{|c|}{ Gefunden: } \\
\hline & & $\mathrm{I}$ & II \\
\hline Baryum & $50,92 \%$ & $51,10 \%$ & $50,82 \%$ \\
\hline Kohlenstoff & 17,84 & 17,68 , & 17,75 \\
\hline Wasserstof & 1,48, & $1,65 \pi$ & 1,91 \\
\hline
\end{tabular}

Die wässerige Lösung des Baryumsalzes wurde mit Silbernitrat gefällt; es schied sich ein weißer Niederschlag aus, der abgesaugt und solange ausgewaschen wurde, bis im ablaufenden Wasser keine Salpetersäure mehr nachzuweisen war. Ein zu. langes Auswaschen bringt großen Substanzverlust mit sich. Das Salz wurde bei $105^{\circ}$ getrocknet, wobei eine Veränderung der Farbe nicht stattfand. Durch Kochen mit Wasser geht es unter Bräunung in Lösung.

Das getrocknete Salz wurde zur Silberbestimmung im Porzellantiegel durch langsames Erhitzen und späteres kräftiges Glühen von den organischen Bestandteilen befreit. Das zurückbleibende metallische Silber wurde als solches gewogen.

$0,2088 \mathrm{~g}$ Substanz lieferten $0,1296 \mathrm{~g}=62,07 \%$ Silber.

$$
\begin{aligned}
& \text { Berechnet für: Gefunden: } \\
& \mathrm{C}_{4} \mathrm{H}_{4} \mathrm{O}_{5} \cdot \mathrm{Ag}_{2} \\
& \text { Silber } \quad 62,06 \% \quad 62,07 \%
\end{aligned}
$$

Es stimmte demnach auch dieses Salz auf Äpfelsaures Silber, nicht nur in seinem Silbergehalt, sondern auch in seinen Eigenschaften.

\section{Die freie Säure.}

Versuche, die freie Säure in krystallisiertem Zustande zu gewinnen, blieben, wie schon oben hervorgehoben wurde, ohne Erfolg. In wässeriger Lösung dreht die Säure die Ebene des polarisierten Lichtes nicht. Sie reduziert Palladiumchlorür-Lösung beim Kochen, Auch in linksdrehenden Blütenhonigen findet sich die gleiche Säure, so daß sie als normaler Bestandteil aller Honige anzusehen ist.

\section{Darstellung der Honigdextrine.}

Da die Fällungen mit Methylalkohol nicht den gesuchten rechtsdrehenden Körper des Koniferenhonigs ergeben, sondern hauptsächlich Phosphorsauren und Äpfelsauren Kalk neben stickstoffhaltiger Substanz niedergeschlagen hatten, wurden nunmehr Versuche angestellt, ihn auf andere Weise zu isolieren.

Fällungen mit $96^{\circ} / 0$-igem Äthylalkohol ergaben immer nur schmierige Ausscheidungen, auch wenn diese wiederholt in wenig Wasser gelöst und in gleicher Weise nocbmals gefällt wurden. Es wurden deshalb Versuche angestellt, den Honig mit Mischungen von Methyl- und Äthylalkohol zu fällen, und zwar unter verschiedenen. Mischungsverhältnissen der beiden Alkohole. Bei Zusatz von viel Methyl- und wenig Äthylalkohol erhielt man wohl flockige Ausscheidungen, aber es blieb in diesem Falle zu viel der rechtsdrehenden Substanz in Lösung. Umgekehrt entstanden wieder mit wenig Methyl. und viel Äthylalkohol Schmieren. Auf Grund vieler Versuche wurden schließlich die günetigsten Fällungsbedingungen gewäblt, die einerseits zu einer möglichst guten Ausbeute, andererseits zu flockigen Ausscheidungen führten, denn offenbar werden klebrige, schmierige Massen immer Zucker mitreißen. Bemerkt sei noch, bevor 
ich näher auf die Methode selbst eingehe, daß man gezwungen ist, nicht mehr als $100 \mathrm{~g}$ Honig auf einmal zur Fällung zu bringen, denn sonst entstehen auch bei dieser Methode leicht Schmieren, dagegen bei genauer Einhaltung aller Verhältnisse wenigstens bei der zweiten Fällung schon flockige Ausscheidungen. Dann mußte natürlich auch das Augenmerk darauf gerichtet sein, den Körper möglichst frei von Farbstoff zu gewinnen, da sonst die Feststellung der spezifischen Drehung unmöglich ist, ganz abgesehen davon, daß die Reinheit der Fällungen sehr beeinträchtigt würde.

Merkwürdigerweise gelingt ein Entfärben der wässerigen Lösung des mehrmals gefällten Körpers nicht so gut, als wenn man zuerst den Honig in wässeriger Lösung durch einmaliges Aufkochen mit ausgeglühter Tierkohle entfärbt, zur früheren Honigkonsistenz eindampft und dann erst zur eigentlichen Fällung schreitet, Auf diese Weise entfärbter Honig wurde nun unter Beobachtung folgender Mischungsverhältnisse etc. zur Fällung verwendet:

$100 \mathrm{~g}$ Honig werden durch Erwärmen verflüssigt und mit $200 \mathrm{ccm}$ Methylalkohol angerieben. Die Lösung bleibt zunächst klar, aber nach einigem Stehen bilden sich flockige Ausscheidungen, die, wie vorher bewiesen, aus Phosphorsaurem und Äpfelsaurem Kalk bezw. Magnesia und stickstoffhaltiger Substanz bestehen.

Von diesen wird nach 24-stündigem Stehen abfiltriert und zum Filtrat $700 \mathrm{ccm}$ Äthylalkohol $(96 \%)$ unter Unschütteln allmählich hinzugesetzt. Es entsteht eine fast weiße Ausscheidung in Flocken, die sich aber bei dieser ersten Fällung durch den Wassergehalt des Honigs bald an der Gefäßwand festsetzen. Die Flüssigkeit wird nach kurzem. Stehen abgegossen; läßt man die Ausscheidung länger mit derselben in Berührung, dann geht wieder ein Teil davon in Lösung. Verfährt man mit der ganzen Operation rasch, so bleibt nur ein kleiner Teil des rechtsdrehenden gesuchten Körpers in Lösung, wie später durch einen besonderen quantitativen Versuch bewiesen werden wird. Die an der Gefäßwand festhaftende Ausscheidung wird in $15 \mathrm{~cm}$ Wasser gelöst, mit $15 \mathrm{cem}$ Methylalkohol verdünnt, filtriert und zu einer Mischung von $200 \mathrm{ccm}$ Methylalkohol und $800 \mathrm{ccm}$ Äthylalkohol hinzugefügt. Schon bei dieser zweiten Fällung bildet sich eine rein weiße, flockige Ausscheidung, die sich auch bei einigem Stehen nicht mehr zu einer klebrigen Masse festsetzt. Der geringe Zusatz von Methylalkohol zu der wässerigen Lösung vor der Fällung hat den Zweck, erstens die stickstoffhaltige, noch in Lösung befindliche Substanz vollständig auszufällen und zweitens, damit die Lösung in verdünnterem Zustand wieder zur Fällung gebracht: wird. Unterläßt man diesen Zusatz, so bilden sich leicht klebrige Ausscheidungen, die sich zusammensetzen und dadurch Zucker einschließen würden. Die Fällung wird nun rasch abgesaugt, wieder in $15 \mathrm{ccm}$ Wasser gelöst und die Lösung nach Zusatz von $15 \mathrm{ccm}$ Methylalkohol abermals in die gleiche Mischung von Methyl- und Äthylalkohol gegeben. Nach dem Absaugen und Auswaschen mit absolutem Alkohol wird die ganze Nutsche mit der Substanz möglichst rasch in einen mit Phosphorpentoxyd beschickten Exsikkator gebracht und im Vakuum getrocknet.

Unterbleibt das Auswaschen mit absolutem Alkohol, so wird die Substanz nach einiger Zeit im Exsikkator infolge zurückgebliebener Feuchtigkeit schmierig.

Auf diese Weise entsteht ein rein weißes, äußerst hygroskopisches Pulver, das in Wasser sehr leicht, klar und fast farblos löslich ist. Die wässerige Lösung reduziert Fehling'sche Lösung nicht sofort, sondern erst nach einigem Aufkochen scheint Hydrolyse durch das Alkali einzutreten und infolgedessen entsteht eine Abscheidung von Kupferoxydul. Dasselbe ist mit Ost'seher Lösung und Soldain i's Reagens der Fall; 
erst nach einigem Kochen tritt geringe Abscheidung von Kupferoxydul ein, die sich bei längerer Kochdauer allmählich vermehrt. Dagegen findet auf Barfoed's Reagens auch bei langem Kochen keine Einwirkung statt.

Auf Grund dieser Erscheinung und unserer Erfahrung glauben wir uns zu der Behauptung berechtigt, daf dieses Honigdextrin an sich nicht reduziert, daß es dagegen durch Alkali leicht hydrolysiert wird, woduich dann Reduktion zu Kupferoxydul eintritt.

Leider erwies sich der Körper als nicht aschenfrei; er enthielt noch 10-15\% Asche, die namentlich aus phosphorsauren Salzen bestand.

Es wurden folgende Versuche angestellt, den Aschengehalt möglichst zu entfernen:

$5 \mathrm{~g}$ des aschenhaltigen Dextrins wurden mit Methylalkohol angerieben und mit $500 \mathrm{~g}$ desselben Alkohols stehen gelassen. Der größere Teil der Substanz löste sich nach einiger Zeit auf, und ich hoffte, daß gerade der ungelöst bleibende Rückstand die Mineralbestandteile enthalten müßte. Die Lösung wurde von dem Rückstand abfiltriert, $400 \mathrm{ccm}$ Methylalkohol abdestilliert und durch $400 \mathrm{ccm}$ Äthylalkohol ersetzt. Es schied sich der Körper in weißen Flocken ab, aber eine Aschenbestimmung der wie vorher getrockneten Substanz zeigte nur eine geringe Abnahme der Mineralbestandteile.

Nach mannigfachen anderen Versucken, den Aschengehalt zu vermindern, so z. B. durch wiederholtes Lösen und Fällen des Dextrins, wurden schließlich durch folgendes Verfahren bessere Ergebnisse erzielt, weshalb jedes, wie vorher, dargestellte Dextrin von nun ab immer dieser Reinigung unterzogen wurde:

$5 \mathrm{~g}$ Substanz werden in $1000 \mathrm{ccm}$ Methylalkohol gelöst, die Lösung mit 50 Tropfen Salzsäure (spez. Gew. 1,125) angesäuert und sofort mit $500 \mathrm{ccm}$ Äther versetzt, da in saurer Lösung Äthylalkohol nicht mehr im stande ist, das Dextrin auszufällen. Die durch den Zusatz von Äther erfolgte Ausscheidung wird möglichst rasch abgesaugt und mit absolutem Alkohol so lange ausgewaschen, bis die ablaufende Flüssigieit neutral reagiert. Die Dextrine halten leicht Salzsäure zurück, deshalb muß auf deren vollständige Entfernung durch Auswaschen mit absolutem Alkohol besondere Sorgfalt gelegt werden. Ein Trocknen über Phosphorpentoxyd gelingt sicherer als über Schwefelsäure.

Auf diese Weise wurde ein rein weißes, sehr leichtes Pulver erhalten, fast farblos und äußerst leicht in Wasser löslich, schwerer, aber vollkommen in Methylalkohol. Es hat wenig süßen Geschmack und ist äußerst bygroskopisch. Das so erhaltene Dextrin muß längere Zeit im Wasserstoffstrome bei einer Temperatur von $100^{\circ}$ bis zur Gewichtsbeständigkeit getrocknet werden, da diese Körper die merkwürdige Eigenschaft haben, größere Mengen Alkohol lange zurückzuhalten. Ein über Phosphorpentoxyd lange Zeit getrocknetes Dextrin verlor nämlich beim Trocknen im Wasserstoffstrome bei $100^{\circ}$ noch $5 \%$ seines Gewichtes.

Eine Aschenbestimmung zeigte nun folgende Zahlen:

$1 \mathrm{~g}$ Dextrin lieferte einen Rückstand von $0,0247 \mathrm{~g}=2,47 \%$ Asche.

Ein Versuch durch nochmalige Fällung des Körpers in saurer Lösung den Aschengehalt weiter herabzudrücken, gelang nur in sebr beschränktem Maße; nach dieser fünften Fällung wurden die Mineralbestandteile auf 2,26\% vermindert. Deshalb wurde davon abgesehen; denn einerseits wurde der Aschengehalt zu wenig vermindert und andererseits ging dabei zu viel an Substanz verloren. Wohl kann man durch Fällen mit mehr Äther eine bessere Ausbeute erzielen, dafür erhält man aber wieder einen Körper, der mehr Mineralbestandteile enthält. 
Bestimmung der spezifischen Drehung.

$1,3215 \mathrm{~g}$ aschenfreie Substanz, (die 2,47\% Asche wurden in Abzug gebracht) wurden in $25 \mathrm{ccm}$ Wasser gelöst und diese Lösung im $200 \mathrm{~mm}-\mathrm{Rohr}$ polarisiert. Sie zeigte eine Rechtsdrehung von $16,5^{\circ}$. Danach beträgt die spezifische Drehung:

$$
[\alpha]_{D}=-\frac{16,5 \cdot 25}{1,3215 \cdot 2}=+156^{\circ}
$$

Ein aus demselben Honig dargestelltes zweites Präparat, bei dem aber eine Fällung mehr vorgenommen war, wurde ebenfalls auf seine spezifische Drehung untersucht.

$0,9283 \mathrm{~g}$ Substanz wurden in $20 \mathrm{ccm}$ Wasser gelöst und im $200 \mathrm{~mm}$-Rohr polarisiert; die Drehung betrug $+14,58^{\circ}$, also die spezifische Drehung:

$$
[\alpha]_{D}=\frac{14,58 \cdot 20}{0,9283.2}=+157^{0}
$$

Dieses ziemlich genaue Übereinstimmen der spezifischen Drehungen bei ganz getrennt isolierten Dextrinen, einmal durch viermalige, das andere Mal durch fünfmalige Fällung, zeigt, daß hier höchstwahrscheinlich eine einheitliche Substanz vorliegt. Auch bei wiederholter Neudarstellung wurde immer ein Dextrin von konstanter spezifischer Drebung erhalten.

\section{Hydrolyse des Dextrins.}

$20 \mathrm{ccm}$ einer wässerigen Lösung, die im $200 \mathrm{~mm} \cdot$ Rohr eine Rechtsdrehung von $16,5^{\circ}$ zeigte, wurden mit $2 \mathrm{ccm}$ Salzsäure (spez. Gew. 1,125) invertiert, und zwar wurde diese Inversion so ausgeführt, daß die Lösung mit der angegebenen Menge Salzsäure in einen Soxhlet-Kolben gebracht und am Rückflußkühler in siedenden Wasserbade eine Slunde erwärmt wurde. Nach Verlauf dieser Zeit war die Rechtsdrehung auf $4,86^{\circ}$ zurückgegangen, die durch weiteres Erwärmen unter den obigen Bedingungen nicht weiter vermindert wurde.

Diese konstant bleibende Drehung, auf Glykose berechnet, zeigt, daß 1 g Dextrin bei der Inversion $0,8716 \mathrm{~g}$ Glykose liefert. Auch der Reduktionswert einer invertierten und entsprechend verdünnten Lösung stimmt genau auf Glykose, ein Beweis, $\mathrm{da} B$ bei der Inversion nur Glykose gebildet worden ist. $25 \mathrm{ccm}$ einer Lösung, die nach der Polarisation 0,1842 $\mathrm{g}$ Glykose enthält, liefert mit $50 \mathrm{ccm}$ Feh ling' scher Lösung bei einer Kochdauer von zwei Minuten 0,3498 g Kupfer, welches 0,1843 g Glykose entspricht.

Wohl ist die Menge der durch Inversion gebildeten Glykose (1 g Dextrin $=0,8716 \mathrm{~g}$ Glykose) zu gering, es konnte aber sein, daß die zur Inversion verwendeten $10 \%$ Salzsäure (spez. Gew. 1,125) bereits gebildete Glykose zerstört hatten. Es wurde deshalb der Versuch gemacht, eine Hydrolyse des Dextrins mit der Hälfte Salzsäure auszuführen. Hierdurch wurde ein etwas besseres Ergebnis erzielt, aber immerhin blieb noch ein Fehlbetrag an Glykose.

$20 \mathrm{ccm}$ Lösung wurden mit $1 \mathrm{ccm}$ Salzsäure (spez. Gew. 1,125), wie vorber, im siedenden Wasserbade invertiext. Die Rechtsdrehung, die vor der Inversion 14,58 betragen hatte, ging im Laufe einer Stunde auf $4,44^{\circ}$ zurück; ausgerechnet auf Glykose würde demnach $1 \mathrm{~g}$ Dextrin $0,9069 \mathrm{~g}$ Glykose bilden. Nach dem Reduktionswert gegenüber Febling'scher Lösung lieferte $1 \mathrm{~g}$ Substanz hierbei $0,9080 \mathrm{~g}$ Glykose. 
Da auch diese Hydrolyse mit 5\% Salzsäure einen Fehlbetrag an Glykose aufwies, wurden Inversionsversuche mit schwächeren Säuren gemacht. Dabei zeigte sich aber, daß das Dextrin äußerst widerstandsfähig gegen diese Säuren war. Essigsäure wirkt überhaupt nicht hydrolysierend; selbst durch Erhitzen mit $0,5 \%$ Oxalsäure unter Druck während zwei Stunden gelingt keine Inversion. Erst 2\% Oxalsäure wirken unter Druck langsam hydrolysierend. Eine Lösung, die im $200 \mathrm{~mm}$-Rohr $+12,25^{0}$ drehte, wurde mit $2^{\%} / 0$ Oxalsäure unter Druck gesetzt, dabei ging die Rechtsdrehung in 2 Stunden auf $6,5^{\circ}$ zurück. Erst nach sechsstündigem Erhitzen im Wasserbade unter Druck betrug die Rechtsdrehung 5,7 ${ }^{\circ}$. Bei diesem Standpunkt wurde der Reduktionswert gegen Fehling'sche Lösung bestimmt, dabei stellte sich aber heraus, daß noch keine vollständige Inversion eingetreten war, denn $1 \mathrm{~g}$ Substanz ergab nur $0,65 \mathrm{~g}$ Glykose. Selbst $0,5 \%$ Schwefelsäure hydrolysiert dieses Dextrin in 2 Stunden nur unvollkommen; es ergab dabei $1 \mathrm{~g}$ Substanz nur 0,55 $\mathrm{g}$ Glykose.

\section{Prüfung auf Furfurolbildung.}

Auch dieser Versuch wurde ausgeführt, weil die nach der Inversion gefundene Menge an Glykose zu gering war. Es lag die Vermutung nahe, daß dieser Mindergehalt an gefundener Glykose durch eine Furfurolbildung erklärt werden könnte. Es wurde zu diesem Zweck $1 \mathrm{~g}$ Substanz mit $20 \mathrm{ccm}$ Salzsäure (spez. Gew. 1,06) in einem Bade von Rose'schem Metall destilliert. Dureh einen Tropftrichter wurde Salzsäure von demselben spez. Gewichte in demselben Maße zufließen gelassen, als solche abdestillierte. Das Destillat wurde auf Furfurol geprüft, indem ein Tropfen desselben auf mit Anilinacetat-Lösung getränktes Filtrierpapier gebracht wurde. Nach dem Abtrocknen zeigte sich eine ganz scbwache Rotfärbung, so daß kein Beweis für Furfurolbildung erbracht werden konnte.

\section{Versuch der Darstellung eines Osazons.}

Alle Versuche, mit dem isolierten Dextrin ein Osazon darzustellen, blieben erfolglos. Sowohl die Darstellung eines Osazons mit $\beta$-Naphtylhydrazin in methylalkoholischer Lösung, als auch mit Essigsaurem Phenylhydrazin in wässeriger Lösung, führte zu keinem Ergebnisse. Dab es gelingt, mit dem hydrolysierten Körper ein Osazon zu erhalten, ist klar, aber trotzdem wurde es dargestellt, als weiterer Beweis, daß bei der Hydrolyse Glykose entstanden war. Zu diesem Zwecke wurde $1 \mathrm{~g}$ Dextrin in $20 \mathrm{ccm}$ Wasser gelöst, und diese Lösung mit $1 \mathrm{ccm}$ Salzsäure (spez. Gew. 1,125) invertiert. Nach Verlauf einer Stunde ist, wie vorher bewiesen wurde, vollständige Hydrolyse eingetreten. $\mathrm{Zu}$ der erkalteten und neutralisierten Lösung wurden $2 \mathrm{~g}$ Phenylhydrazinhydrochlorid und $3 \mathrm{~g}$ Natriumacetat hinzugefügt und auf dem Wasserbade erwärmt, indem man zur $\Delta$ bhaltung der Luft einen kräftigen Strom Kohlensäure einleitete. Nach kurzer Zeit sehied sich das Osazon aus. Es hatte nach wiederholtem Umkrystallisieren den konstanten Schmelzpunkt von 204\%. Es liegt also hier Phenylglykosazon vor, ein Beweis, daß bei der Hydrolyse Glykose entstanden war.

\section{Elementaranalyse.}

Diese erbringt den Beweis, daß in diesem Dextrin wirklich ein Polysaccharid der empirischen Formel $\mathrm{C}_{6} \mathrm{H}_{10} \mathrm{O}_{5}$ vorliegt. Vor der Verbrennung mußte besonders 
darauf geachtet werden, daß die Substanz bis zur Gewichtsbeständigkeit getrocknet wurde, denn die letzten Anteile Feuchtigkeit scheinen erst nach langem Erhitzen im Wasserstoffstrome fortzugehen. Die besten Ergebnisse wurden erzielt, wenn die Sub$\operatorname{stanz}$ in demselben Schiffchen verbrannt wurde, in dem sie bis zur Gewichtsbeständigkeit getrocknet war; es wurden folgende Zahlen erhalten:

1. $0,2294 \mathrm{~g}$ (aschenfreie) Substanz lieferten $0,3720 \mathrm{~g}$ Kohlensäure und $0,1380 \mathrm{~g}$ Wasser $=44,23 \%$ Kohlenstoff und $6,72 \%$ Wasserstoff.

2. $0,2548 \mathrm{~g}$ (aschenfreie) Substanz lieferten $0,4140 \mathrm{~g}$ Kohlensäure und $0,1500 \mathrm{~g}$ Wasser $=44,35 \%$ Kohlenstoff und $6,59 \%$ Wasserstoff.

\begin{tabular}{|c|c|c|c|}
\hline \multirow{2}{*}{\multicolumn{2}{|c|}{$\begin{array}{l}\text { Berechnet für: } \\
\qquad\left(\mathrm{C}_{6} \mathrm{H}_{10} \mathrm{O}_{5}\right) \times\end{array}$}} & \multicolumn{2}{|c|}{ Gefunden: } \\
\hline & & I & III \\
\hline $\mathrm{hl}$ & . . $44,44 \%$ & $44,23 \%$ & $44,35 \%$ \\
\hline Wassersto & 6,17 & 6,72 & 6,59 \\
\hline
\end{tabular}

Der Honig, aus dem dieses Dextrin abgeschieden wurde, stammte aus dem badischen Schwarzwald; seine spezifische Drehung war $+16,8^{\circ}$. Nachdem das Dextrin nach der oben angegebenen Methode gefällt war, betrug seine Drehung $+4,16^{\circ}$. Ein Beweis, dab ein großer Teil dieser nach der Fällung verbleibenden Rechtsdrehung durch die Anwesenheit von Saccharose hervorgerufen wurde, konnte in diesem Falle dadurch erbracht werden, daß mit $0,5 \%$ Oxalsäure auf dem Wasserbade bei $70^{\circ}$ invertiert wurde. Das in diesem Honig enthaltene Dextrin wird ja dadurch nicht angegriffen; durch die Hydrolyse der Saccharose ging die spezifische Drehung des Honigs auf $-1,4^{0}$ zurück.

Ich hoffte nun, durch diese Methode ein Dextrin isoliert zu haben, welches in jedem Koniferenhonig in gleicher Zusammensetzung, mit gleicher spezifischer Drehung und gleichen Eigenschaften vorkommt. Aber sofort der zweite zur Untersuchung gelangte Honig lieferte ein Dextrin von anderer spezifischer Drehung und von zum Teil anderen Eigenschaften.

Auf Grund dieser verschiedenen Ergebnisse wurden auch Dextrine aus drei weiteren Koniferenhonigen nach derselben Methode dargestellt. Aus jedem Honig wurde ein Körper von konstanter spezifischer Drehung erhalten, ob er vier- oder fünfmal gefällt wurde. Jedes der drei folgenden Dextrine ergab eine andere spezifische Drehung.

Es darf daher angenommen werden, daß in jedem Koniferenhonig eine Übergangsverbindung zwischen Stärke und Zucker vorhanden ist, das sich leicht durch die angegebene Fällungsmethode als einheitliche Substanz gewinnen läßt. Die folgende Tabelle zeigt die Eigenschaften der dargestellten Dextrine:

\begin{tabular}{|c|c|c|c|c|c|c|}
\hline \multirow{2}{*}{ No. } & \multirow{2}{*}{$\begin{array}{l}\text { Herkunft und Jahrgang } \\
\text { des Koniferenhonigs }\end{array}$} & \multicolumn{2}{|c|}{ Spez. Drehung $[\alpha]_{D}$} & \multirow{2}{*}{$\begin{array}{l}\text { Verhältnis der } \\
\text { Drehungswinke] } \\
\text { einer 50\% } 5 \text {-igen } \\
\text { Iosung vor } \\
\text { und naeh der } \\
\text { Inversion }\end{array}$} & \multicolumn{2}{|c|}{$\begin{array}{c}\text { 1. g Dextrin liefert bei } \\
\text { der Inversion } \\
\text { Glyk os }\end{array}$} \\
\hline & & $\begin{array}{c}\text { des } \\
\text { Honigs }\end{array}$ & $\begin{array}{c}\text { des } \\
\text { Dextrins }\end{array}$ & & $\mid \begin{array}{c}\text { nach der } \\
\text { Polarisation }\end{array}$ & $\begin{array}{l}\text { nach dem } \\
\text { Reduktions- } \\
\text { wert }\end{array}$ \\
\hline 1 & Baden, Kinzigtal (1902) & $+16,870$ & $+157,00^{0}$ & $1: 0,2945$ & $0,8716 \mathrm{~g}$ & $0,8720 \mathrm{~g}$ \\
\hline 2 & Baden, Murgtal (1903) & $+7,90^{\circ}$ & $+131,28^{0}$ & $1: 0,3830$ & 0,8297 & 0,9080 \\
\hline 3 & Oberelsak (1908) & $+12,070$ & $+125,590$ & $1: 0,3000$ & 0,7144 & 0,8362 , \\
\hline 4 & Wasserburg am Bodensee (1903) & $+8,500$ & $+119,90^{\circ}$ & $1: 0,3047$ & 0,6920 & 0,8249 \\
\hline
\end{tabular}

Diese Tabelle zeigt folgendes: 
Bei allen Dextrinen geht die Rechtsdrehung durch Inversion mit Salzsäure stark zurück, und zwar ist der Unterschied der Drehungswinkel vor und nach der Inversion bei Dextrin aus dem Honig 1 mit der spezifischen Drehung $+157^{\circ}$ am größten. Auffallend ist, daß die bleibende Rechtsdrehung bei diesen Dextrinen genau auf Glykose stimmt, während bei den übrigen drei Körpern der Reduktionswert höher ist als der nach der Inversion bleibenden Rechtsdrehung entspricht. Es scheint jedoch hier eine gewisse Gesetzmäßigkeit zu herrschen, insofern als der Unterschied der Rechtsdrehung und des Reduktionswertes um so gröber ist, je niedriger das spezifische Drehungsvermögen des Dextrins ist. In ihren Eigenschaften ähneln sich die letzten drei Dextrine sehr. Diese wurden zum Unterschied von dem Dextrin mit $[\alpha]_{D}=$ $+157^{\circ}$ leichter hydrolysiert; so greift schon $0,5 \%$ Oxalsäure, wenn auch langsam, hydrolysierend an. Dagegen vermag verdünnte Essigsäure auch hier keine Inversion herbeizuführen. In Methylalkohol sind sie leichter löslich als das Dextrin mit $[\alpha]_{D}$ $=+157^{\circ}$.

Ein durchschlagender Unterschied der drei Dextrine von dem Dextrin $[\alpha]_{D}=$ $+157^{\circ}$ ist auch der, daß sie bei längerem Kochen mit Essigsaurem Phenylhydrazin in wässeriger Lösung ein Osazon bilden, das sich als Phenylglycosazon erwies.

\section{Gärungsversuche.}

O. Künumann und A. Hilger haben das von ihnen isolierte Honigdextrin auf seine Vergärbarkeit mit gut charakterisierten, leicht kontrollierbaren Hefenreinkulturen untersucht. Sie kommen zu dem Schluß, daß „Weinhefen im stande sind, das Honigdextrin nur in sehr geringer Menge zu vergären, daß dagegen Bierhefen in allen Fällen bedeutend energischer wirken, aber dennoch böchstens die Hälfte aufzehren und daß nur Schizosaccharomyces Pombe es vollständig vergärt."

Sie stellten sich sogenannte Normalgärlösungen her, die, außer einem bekannten Gebalt an Dextrin, Fruktose und Glykose in einem Verhältnisse enthielten, wie diese im Naturhonig vorkommen.

Bei dieser Methode wäre es denkbar, daß das Honigdextrin allein nicht vergären würde, daß es nur durch die Vergärung der beiden Zuckerarten mit in diese hereingerissen werden könnte. Dann auch war es so unmöglich, festzustellen, ob Vergärung oder Assimilation des Dextrins stattfindet.

Unser Augenmerk war daher darauf gerichtet, Dextrinlösungen in Hefenwasser als Nährlösung ohne Zusatz von Zucker auf Vergärbarkeit zu prüfen; auch wurde eine andere Methode angewendet, und zwar die von P. Lindner ausgearbeitete, die es ermöglicht, mit wenig Substanz viele Hefen durchzuprobieren.

Die Versuche wurden sämtlich in der Wissenschaftlichen Station für Brauerei in München (Physiologisch-biologische Abteilung) ausgeführt, und zwar unter der gütigen Aufsicht und Anleitung des Herrn Professor Dr. Will. Der gröBte Teil der untersuchten Hefenreinkulturen stammte aus diesem Institut, einige aus dem Institut für Gärungsgewerbe in Berlin und endlich die Weinhefen aus der Hefenreinzucht-Station bei der Kgl. Lehranstalt für Wein- und Gartenbau in Geisenheim.

Diese Gärungsversuche wurden wesentlich gefördert durch das Entgegenkommen nachstehender Herren, denen wir für ihr Interesse und für die Überlassung von Hefenreinkulturen zu ganz besonderem Dank verpflichtet sind: Herrn Professor Dr. Lintner, Professor an der Technischen Hochschule und Direktor der Wissenschaftlichen Station für Brauerei in München und Herrn Professor Dr. Will, Abteilungsvorsteher des 
genannten Instituts, Herrn Professor Dr. P. Lindner, Vorsteher des botanischen Laboratoriums des Instituts für Gärungsgewerbe in Berlin, Herrn Professor Dr. Wortmann, Direktor der Kgl. Lehranstalt für Wein-, Obst- und Gartenbau in Geisenheim.

Bevor ich zu den Versuchen selbst übergehe, sei kurz beschrieben, wie die Hefenreinkultur bis zur Verwendung zu den Versuchen in zweckentsprechender Weise vorbereitet wurde:

Von der in Bierwürze vermehrten Hefe wurde eine Strichkultur auf gehopfter Würzegelatine angelegt. Die Weinhefen wurden, da Traubenmost nicht zur Verfügung stand, ebenfalls in Bierwürze aufgefrischt, die Strichkultur dagegen hier auf ungehopfter Würzegelatine angelegt. Thre Entwickelung war auch so eine normale. Diese Strichkulturen wurden in den Thermostaten bei $20^{\circ}$ gebracht, und nach 8-14 Tagen hatte sich die Hefe genügend vermehrt, um sie zur Anlegung von Gärversuchen verwenden zu können. $\mathrm{Zu}$ diesem $\mathrm{Zwecke}$ wurden 2-3 Platinösen von dem dicken Hefenbelag abgenommen und in etwa $10 \mathrm{~cm}$ sterilem Hefenwasser möglichst fein verteilt, mit der Vorsicht, daß ein Übertragen von Nährgelatine vermieden wurde. Alle diese Operationen, wie das nachfolgende Anlegen der Versuche, wurden unter Beobachtung der üblichen Vorsichtsmaßregeln im Impfkasten ausgeführt.

Als Gärgefäße dienten nach P. Lindner sterile, hohlgeschliffene Objektträger, die man auf folgende einfache Weise mit der Hefe und der Dextrinlösung beschickt: Um die Höhlung des Objektträgers, deren Durchmesser etwa $1^{1 / 2} \mathrm{~cm}$ beträgt, wird mit einem Pinsel ein etwa $1 / 2 \mathrm{~cm}$ breiter Ring von Vaselinöl gezogen und mit einer sterilen Pipette 2--3 Tropfen sterilen Hefenwassers, in dem die betreffende Hefe vorher fein verteilt war, in die Höhlung gebracht. Mit einem vorne breit geklopften Platindraht wird nun eine kleine Menge Dextrin in das Hefenwasser auf den Objektträger gegeben und durch Umrühren mit dem Platindraht in Lösung gebracht. Dann wird die Höblung mit einem Deckgläschen durch Darüberschieben verschlossen, mit der Vorsicht, daß keine Luftblase darunter verbleibt. Ein etwaiges Zuviel der Flüssigkeit muß mit sterilem Filtrierpapier sorgfältig entfernt werden, da sonst der Vaselinring nicht dicht schließen würde.

Das so hergestellte Präparat bringt man in den Thermostaten von $20^{\circ}$. Nach 24-, spätestens 48-stündigem Stehen zeigt sich, ob eine Gasentwickelung eingetreten ist oder nicht. In letzterem Falle erscheint das Präparat unverändert; ist eine Gärung eingetreten, so hat sich unter dem Deckglase eine mehr oder weniger große Gasblase gebildet, je nachdem ob das Dextrin stark oder schwach angegriffen worden ist. Zur Vorsicht wurden alle Versuche während drei Tagen im Thermostaten gelassen und täglich beobachtet.

Am praktischsten ist es, wenn man bei jeder Versuchsreihe gleichzeitig mehrere - in unserem Falle 10 - Objektträger auf die angegebene Weise mit Hefe und Hefewasser beschickt und dann je zwei zur Kontrolle ohne Dextrin und die übrigen zu den vier Dextrinen verwendet. Die Kontrolle ohne Dextrinzusatz hat den Zweck, den Nachweis zu erbringen, daß im Hefenwasser selbst vergärbare Substanz fehlt und dann, daß keine Näbrgelatine mit übertragen worden ist, denn dann würde obnehin eine Gärung stattfinden. Die beiden Parallelversuche eines jeden Dextrins müssen nach 24-48 Stunden genau übereinstimmen, im anderen Falle und auch, wenn keine Gärung eingetreten war, wurde der Versuch mit einer frischen Strichkultur wiederholt. Nach unserer Erfahrung kommt es auch sehr neben dem genauen Einhalten der von $P$. Lindner angegebenen Methode darauf an, daf nur eine kleine Menge Dextrin in 
1. Hefenreinkultaren aus der wissenschaftlichen Station für Brauerei in Mü $\mathrm{nchen}$.

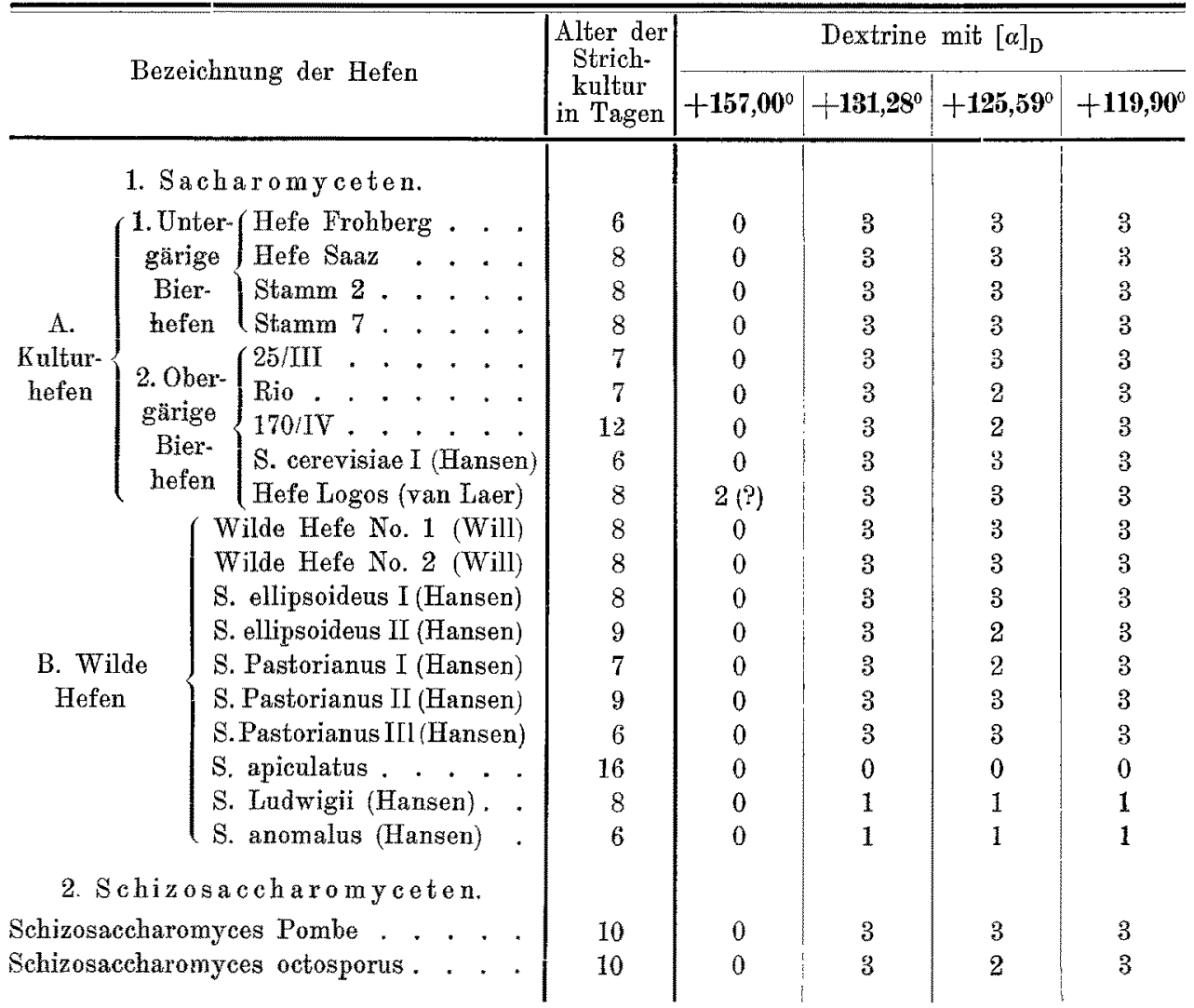

II. Hefenreinkulturen aus dem Institut für Gärungsgewerbe in Berlin.

No. 97, Untergärige Brauereihefe . . . 9

No. 429. Hefe Logos. . . . . . . . 9

No. 487, Rasse V, Preß̧hefe . . . . . 9

No. 574, Rasse VI, Prefhefe . . . . 9

No. 637, Rasse VIII, Preßihefe . . . . 9

No. 578, Weinhefe

\begin{tabular}{l|l} 
& 0 \\
9 & 0 \\
9 & 0 \\
9 & 0 \\
& 0
\end{tabular}

3
3
3
3
3
3

\begin{tabular}{l|l}
3 & 3 \\
3 & 3 \\
3 & 3 \\
3 & 3 \\
3 & 3 \\
3 & 3
\end{tabular}

III. Hefenreinkulturen aus der Hefenreinzuchtstation der Kgl. Lehranstalt für Wein-, Obst-und Gartenbau in Geisenheim.

WeinSteinberg 1892 . . . . . 10

Steinberg 1898

10
10

Akmannshausen ..... 10

Zeltingen . . . . . . 10

hefen

Forst 
[Fortsetzung von S. 122.]

die Höhlung des Objektträgers gebracht wird; denn leicht wird bei der kleinen Flüssigkeitsmenge die Lösung zu konzentriert, und es würde schon deshalb eine Gärung unterbleiben. In vorstehender Tabelle (S. 123) ist der Grad der Gärung durch folgende Zeichen angedeutet: "0" bedeutet, daß keine Gärung eingetreten ist, ", "eine schwache, "2" eine mäbige, „3" eine starke Gärung. Bei letzterer ist oft die ganze Höhlung des Objektträgers vón einer großen Gasblase angefüllt oder gar das Deckgläschen fortgeschoben worden.

Aus der Tabelle ersieht man, daß das Dextrin mit der spezifischen Drehung $[\alpha]_{D}=+157^{\circ}$, außer in einem-zweifelhaften Falle, von keiner Bi erhefe vergoren worden ist. Eine Gasblase wurde bei Hefe Logos (van Laer), und zwar nur bei einem einzigen Präparat dieses Dextrins wahrgenommen. Eine öfters wiederholte Kontrolle mit einer jedesmal frisch angelegten. Strichkultur führte jedoch niemals zu einer Bestätigung der vorigen Beobachtung, so daß man eigentlich gezwungen ist, irgend einen Zufall oder Irrtum und keine Vergärung anzunehmen.

Die übrigen drei Dextrine wurden von allen Hefen, auBer von Saccharomyces apiculatus unter Gasentwickelung angegriffen.

Die Versuche mit Weinhefen lieferten im wesentlichen dasselbe Ergebnis, nur daß überall eine weit schwächere Gasentwickelung, also Vergärung, wahrgenommen wurde. Auch hier wurde das Dextrin mit $[\alpha]_{D}=+157^{\circ}$ in keinem Falle unter Gasentwickelung angegriffen. Auch bei dem Dexirin $[\alpha]_{\mathrm{D}}=+125,59^{\circ}$ konnte bei einigen Hefen keine Gasblase wahrgenommen werden, vielleicht ist aber dort die Kohlensäureentwickelung so gering gewesen, daß dieselbe in der Flüssigkeit gelöst blieb.

Mit einer kräftig wirkenden Hefe, Oberkefe 25/III, wurde nun ein größerer Versuch mit Lösungen von polarimetrisch festgestelltem Dextringehalt angestellt, einerseits um festzustellen, ob das Dextrin $[\alpha]_{D}=+157^{\circ}$ auch nicht zum Aufbau der Hefenzellen assimiliert würde, andererseits, ob die drei übrigen Dextrine vollständig vergoren werden.

Hierzu wurde zunächst die Oberhefe 25/IIr, in einer sterilen Hefe-Zuckerwasserlösung (6 g Rohrzucker in $100 \mathrm{ccm}$ Hefenwasser) vermehrt, bis jede Spur von Saccharose vergoren war. Zur vollständigen Vergärung derselben und zur genügenden Vermehrung der Hefe wurde die in einem Pasteur-Kolben befindliche Lösung während 10 Tagen bei $25^{\circ}$ im Thermostaten belassen. Nach 14 Tagen war die stürmische Hauptgärung vorüber, dann fing die Hefe an, sich zu Boden zu setzen. Von diesem Zeitpunkt ab war es nötig, durch Schwenken des Kolbens die Hefe täglich aufauschütteln, da sonst die unteren Schichten der Hefenzellen von der Nahrung ausgeschlossen werden konnten. Diese Oberhefe $25 / \mathrm{III}$ war also im stande, innerhalb 10 Tagen die Saccharose vollständig zu vergären, deren Abwesenheit sowohl durch Kochen mit Fehling'scher Lösung als auch durch das Polarisationsverfahren festgestellt wurde. Es fand weder eine Reduktion noch eine Ablenkung des polarisierten Lichtstrahles statt.

In der Zwischenzeit wurden Lösungen der vier Dextrine in Hefenwasser hergestellt, deren Gehalt durch die Polarisation ermittelt wurde. Je $25 \mathrm{ccm}$ dieser Lösungen wurden in ein kleines Pasteur-Kölbchen gebracht und im strömenden Dampf zweimal je 10 Minuten an zwei verschiedenen Tagen sterilisiert. Trotz der kurz bemessenen Sterilisation bräunten sich die vorber schwach gelb gefärbten Lösungen etwas, woraus auf eine Karamelisierung von einer Spur Dextrin gesehlossen wurde. In die sterilen Lösungen wurden, unter Vermeidung einer sekundären Infektion, also 
im Impfkasten, je 2-3 Tropfen des dickbreiigen Hefenabsatzes übergeimpft und die Kölbchen in den Thermostaten von $25^{\circ}$ gebracht. In allen vermehrte sich die Hefe deutlich, auch in der Lösung des Dextrins mit $[\alpha]_{D}=+157^{\circ}$, wenn auch schwächer. Bei den drei anderen Dextrinen traten auBerdem Gärungserscheinungen auf, wie solches auch der Vorversuch auf den Objektträgern gezeigt hatte. Die Lösungen wurden so 20 Tage unter täglichem Aufschütteln der Hefe im Thermostaten gelassen, dann von dem Hefenabsatz abfiltriert, bis zum ursprünglichen Volumen von $25 \mathrm{ccm}$ eingedampft und polarisiert. Aus der nachstehenden Tabelle ersieht man die Drehungen der Lösungen vor und nach der Vergärung, und, da die Drehungen proportional dem Gehalt an Dextrin sind, kann man auf eine Abnahme des Dextrins, sei es durch Vergärung oder Assimilation schließen.

\begin{tabular}{|c|c|c|c|c|c|}
\hline \multirow{3}{*}{$\begin{array}{l}\text { Dextrine } \\
\operatorname{mit}[\alpha]_{D}\end{array}$} & \multirow{2}{*}{\multicolumn{2}{|c|}{$\begin{array}{l}\text { Drehungswinkel der Lösung } \\
\text { im } 200 \mathrm{~mm}-\mathrm{Rohr}\end{array}$}} & \multicolumn{3}{|c|}{ Dextringehalt } \\
\hline & & & \multicolumn{2}{|c|}{ in $100 \mathrm{cem}$} & \multirow[b]{2}{*}{ Abnahme } \\
\hline & $\begin{array}{c}\text { vor der } \\
\text { Gärung }\end{array}$ & $\begin{array}{l}\text { nach der } \\
\text { Gärung }\end{array}$ & $\begin{array}{l}\text { vor der } \\
\text { Gärung }\end{array}$ & $\begin{array}{l}\text { naeh der } \\
\text { Gärung }\end{array}$ & \\
\hline$+157,000$ & $+9,803$ & $+8,250$ & $3,121 \mathrm{~g}$ & $2,627 \mathrm{~g}$ & $16 \%$ \\
\hline$+131,280$ & $+9,370$ & $+5,25^{\circ}$ & 3,576 , & 2,003 & $44 \pi$ \\
\hline$+125,590$ & $+9,60^{\circ}$ & $+5,100$ & 3,940 & 2,040 & 47 \\
\hline$+119,900$ & $+9,800$ & $+4,900$ & 4,117 & 2,058 * & 50 \\
\hline
\end{tabular}

Bei dem Dextrin mit $[\alpha]_{D}=+157^{\circ}$ sind die fehlenden $16 \%$ nicht unter Gasentwickelung angegriffen, d. h. vergohren worden, sondern während der 20 Tage zum Aufbau der Hefenzellen assimiliert worden. Denn in der Hefenflüssigkeit war kein Alkohol durch die Jodoform-Reaktion nachweisbar, ebenso war vorher auf den Objektträgern eine Gasentwickelung nicht wahrgenommen worden.

Von den drei anderen Dextrinen sind $44-50 \%$ vergoren worden, und zwar ist der Prozentsatz un so höher, je niedriger das spezifische Drehungsvermögen des Dextrins ist. Aber trotzdem kann man auch diese drei Dextrine noch zu den schwer vergärbaren Substanzen rechnen, da die Oberhefe 25/III, die Saccharose glatt und vollständig vergärt, nicht im stande gewesen ist, sie während 20 Tagen vollständig zu verarbeiten.

Wir kommen auf Grund dieser zahlreichen Versuche zu dem Schluß, daß sämtliche vier Honigdextrime von Gärungsorganismen angegriffen werden und zwar von den untersuchten Weinhefen schwächer als von den übrigen Hefen. Bei dem Dextrin mit $[\alpha]_{D}=+157^{\circ}$ tritt durch keine Hefe Vergärung ein; sehr wahrscheinlich findet jedoch mit allen Hefen eine Assimilation statt, was bei Oberhefe 25/III bestimmt nachgewiesen werden konnte.

\section{Zusammenfassung der Ergebnisse.}

Die Ergebnisse der im vorstehenden beschriebenen Untersuchungen berechtigen folgende Tatsachen festzustellen:

1. Als normaler Bestandteil der Honige, sowohl des Koniforenhonigs, als auch des Blütenhonigs, mub die Apfelsäure betrachtet werden.

2. Die Dextrine des Koniferenhonigs lassen sich durch Fällungen mit Mischungen von Methyl- und Äthylalkohol als einheitliche Körper darstellen. 
3. Dieselben sind als Übergangsstadien von Stärke zu Zucker zu betrachten, und zwar nicht etwa als ein Gemenge von Dextrin und Zucker, sondern als einheitliche Körper vom Charakter der Achroodextrine. Jeder Koniferenhonig enthält ein ihm eigentümliches Dextrin von konstanter spezifischer Drehung, das entweder dem Stärkedextrin oder dem Zucker näher stehen kann. Die Dextrine verschiedener Honige haben ungleiche spezifische Drehung.

4. Isoliert wurde aus vier typischen Koniferenhonigen je ein Dextrin mit folgenden spezifischen Drehungsvermögen $\left([\alpha]_{\mathrm{D}}\right)$ :

$$
+157,00^{\circ} ;+131,28^{\circ} ;+125,59^{\circ} ;+119,900^{\circ} \text {. }
$$

5. Das Dextrin mit $[\alpha]_{D}=+157^{0}$ zeigt typischen Dextrincharakter, besitzt die empirische Formel $\left(\mathrm{O}_{6} \mathrm{H}_{10} \mathrm{O}_{5}\right)$ und ist gegen schwächere Säuren äußerst widerstandsfähig. Nur Salzsäure führt vollständige Inversion herbei.

6. Die übrigen drei Dextrine stehen dem Zucker näher, sie werden durch schwache Säuren leichter hydrolisiert.

7. Alle vier Dextrine reduzieren Fehling'sche und Ost'sche Lösung sowie Soldaini's Reagens nicht; erst nach einigem Kochen tritt Hydrolyse durch das Alkali und infolgedessen Abscheidung von Kupferoxydul ein. Sie wirken auf Barfoed's Reagens auch nach langem Kochen nicht ein.

8. Die Honigdextrine sind nicht im stande, Osazone zu bilden.

9. Das Dextrin mit $[\alpha]_{0}=+157^{\circ}$ wird von kräftig wirkenden Gärungsorganismen nicht unter Gasentwickelung angegriffen. Sehr wahrscheinlich findet jedoch eine Assimilation dieses Dextrins statt, was bei Oberhefe 25/III bestimmt nachgewiesen werden konnte.

10. Die übrigen Dextrine werden dagegen unter Gasentwickelung angegriffen, immerhin gehören sie noch zu den schwer vergärbaren Substanzen.

11. Ober- und untergärige Bierhefen, sowie gewisse Arten von wilden Hefen, aus den Gattungen Saccharomyces und Schizosaccharomyces wirken energischer als die Mehrzahl der untersuchten Weinhefen auf die Honigdextrine ein.

Der Vorsitzende gibt darauf das Ergebnis der Ausschußwahl bekannt, wonach sämtliche bisherigen Ausschußmitglieder wieder gewählt und an Stelle des durch seine Berufung nach Wien ausgeschiedenen Prof. Dr. Prior Dr. A. Bömer-Münster i. W neugewählt wurde.

Rupp macht Mitteilung über den Aschengehalt von Gewürzen und glaubt, daß die vorhandenen Grenzzahlen nicht einwandfrei seien; bei Zimmet sei die Grenzzahl von 5 auf $6 \%$ zu erhöhen. Ebenso seien die Zahlen bei Safran zu erhöhen.

Schlegel erklärt, daß die Gewürzkommission in Bonn grundsätzlich beschlossen habe, die Aschengrenzen bei Gewürzen vorläufig nicht zu erhöhen; die angeregte Frage solle der Gewürzkommission überlassen werden.

Rupp glaubt, daß wir in dieser Beziehung dem Handel entgegenkommen sollten.

Dr. Halenke bestätigt die Ausführungen Rupp's, daß Ceylonzimmet und auch andere Waren höheren Aschengehalt haben können, trotzdem sei heute keine Änderung der Grenzzahlen vorzunehmen, die Beurteilung dieser Waren bleibe ja doch dem Ermessen jedes einzelnen Gutachters überlassen.

Rupp sieht von der Stellung eines Antrages ab. Er macht dann weiter Mitteilung über sogen. Dauerbrot, das 30 Tage unverändert haltbar sein soll und 
zu dessen Herstellung auf $20 \mathrm{~kg}$ Teig $500 \mathrm{~g}$ Sagokleister und $40 \mathrm{~g}$ Natriumphosphat verwendet würden. In dieser Herstellungsweise sei keine Verbesserung zu erblicken und ein Zusatz von Sagokleister sollte als unzulässig erklärt werden.

Dr. Juckenack erklärt, daß außer Sago auch Leguminosenmebl, Reis u. dergI. zur Herstellung von sogen. Dauerbrot verwendet würden; der Zweck werde aber nicht erreicht, die Kruste dieses Brotes werde bald gummiartig und der Geschmack ein altbackener. Mit derartigen Verfahren werde nur den Bäckern das Geld aus der Tasche gelockt und es sei davor zu warnen.

Der Vorsitzende schließt darauf die Versammlung urn $2^{\mathbf{1} / 2}$ Uhr mit Worten herzlichen Dankes an alle, die sich um ihr Gelingen verdient gemacht; insbesondere dankt er den Herren Vortragenden und den Mitgliedern des Ortsausschusses für ihre Mübewaltung und ruft allen Anwesenden ein herzliches "Auf Wiedersehen in Dresden" zu.

Trillich richtet schließlich Worte herzlichen Dankes an den Vorsitzenden für dessen umsichtige Verhandlungsleitung, indem er gleichzeitig der Überzeugung Ausdruck gibt, daß bei einer minder umsichtigen Leitung die teilweise zutage getretenen Gegensätze vielleicht zu weitergehenden Auseinandersetzungen geführt haben würden. Die Versammlung gab diesem Danke dem Vorsitzenden gegrenüber durch Erheben von den Sitzen Ausdruck.

Nach einem zwanglosen Mittagessen im Hotel Viktoria fand am Nachmittag ein Spaziergang zum Waldhause auf dem Hasenberge statt.

Sonntag den 15. Mai 1904 beteiligten sich die noch anwesenden Versammlungsbesucher, einer freundlichen Einladung des Würitembergischen Bezirksvereins des Vereins Deutscher Chemiker folgend, an dessen Ausflug auf den Lichtenstein.

C. Mai.

\section{Gesetze, Gesetz- Entwïre, Verordunngen u. s. w., Gerichts - Entseheidungen.}

\section{Allgemeines.}

Preuben. Erlaß der Ministerien für Handel und Gewerbe, der geistlichen, Unterrichts- und Medizinalangelegenheiten und des Innern. An die Regierungspräsidenten und den Polizeipräsidenten in Berlin. Vom 22. Februar 1904 "Handel und Gewerbe" 1904, 11, 393.) - In neuerer Zeit ist mehrfach darüber Klage geführt worden, daßi die Polizeibehörden bei der Vorbereitung der strafrechtlichen Verfolgung von Verfälschungen von Nahrungsmitteln geeignete Sachverständige nicht in dem erforderlichen Mafe zuzögen. Unter anderem sollen die über die Zusammensetzung der Ware gehörten Chemiker öfter auch als berufene Gutachter über gleichzeitig zu entscheidende auf medizinischem Gebiet oder auf dem Gebiete von Handel und Verkehr liegende Fragen angesehen und es soll von der Anhörung ärztlicher und gewerblicher Sachverständiger Abstand genommen worden sein. Ein solches Verfahren entspricht nicht den bestehenden Bestimmungen. Nach dem unten abgedruckten Erlaß vom 14. September 1883 soll sich die gutachtliche Anhörung der Chemiker auf die Frage der chemischen Zusammensetzung der Ware beschränken und die Begutachtung der weiteren Fragen, $o b$ die Ware in der festgestellten Zusammensetzung gesundheitsschädlich und ob sie "Zum Zweck der Täuschung im Handel und Verkehr" (\$ 10 des Nahrungsmittelgesetzes) ver. falscht ist, ärztlichen bezw. gewerblichen, speziell mit den Gewohnheiten des betreffenden Industriezweiges vertrauten Sachverständigen unterstehen. Die Zuziehung solcher Sachverständiger soll in allen irgendwie zweifelhaften Fällen erfolgen.

Besonderer Wert muk darauf gelegt werden, daß die Polizeibehörden die erforderlichen Gutachten von geeigneter Stelle einholen. Zu dem Ende haben sie für Fragen auf derm Gebiete von Handel und Verkehr die amtlichen Handelsvertretungen um Benennung geeigneter Sachverständiger, geeignetenfalls wn direkte Abgabe eines Gutachtens za ersuchen.

Wir ersuchen Sie, den Polizeibehörden den genannten Erlaß in Erinnerung zu bringen und sie dabei auf die vorgedachten Punkte besonders hinzuw eisen.

Den Handelsvertretungen wird dieser Erlafs direkt mitgeteilt werden. 